\title{
Crack Detection Method of Vibrating Screen Beam Based on Fracture Mechanics
}

\author{
Dong Li, Mingyue Ren, Guoying Meng \\ China University of mining and technology, Beijing 100083, China. \\ tbp130402013@student.cumtb.edu.cn
}

Keywords: Vibrating screen, Beam, Crack detection, Flexibility.

\begin{abstract}
Vibrating screen is one of the important washing equipment in coal washery, and it is mainly used to grading, dielectric recovery, raw coal cleaning, etc. The safe operation of vibrating relates to the coal mine production safety directly. After continuously run for a long time, various faults of vibrating screen cannot be avoided, and beam fracture is one of the most common and serious fault. Now researches for the early detection of beam crack have made some achievements, but many of them can only detect the existence crack, and has not been able to detect the location and depth of crack. In this paper, the crack detection of beam based on fracture mechanics method to solve this problem, so as to realize the quantitative detection of crack. It also can provide the basis for faults predicting of vibrating screen.
\end{abstract}

\section{Introduction}

Fracture failure is a common form of mechanical failure. In a sense, crack can be seen as the early form of fracture failure. So the early detection of crack is of significant important to the safe and reliable operation of mechanical structures. The local flexibility method based on the fracture mechanics is one of the commonly used methods dealing with crack problems.

The vibrating screen, which is one of the most important washing equipment in coal washery, is mainly used to achieve the raw coal classification, medium recovery, etc. There would be some faults appear like screen box crack, beam fracture, bearing failure, etc in vibration screen after continuous operation. Among them, the beam fracture is the most common and serious fault. Beam is one of the key components, and the common structure forms are shown in figure 1.
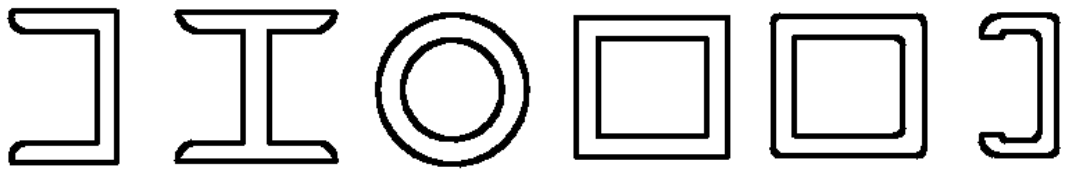

Fig. 1 Common forms of vibrating screen beam

For the time being, the research on crack detection of vibrating screen beam is limited in quantity. However, few researches can detect the crack of beam quantificationally. The change in structure dynamic response because of the crack is the basis of quantitative detection, and it attracts much attention of researchers all over the world. Dimarogonas combined fracture mechanics with rotor dynamics to deal with the crack detection in rotors. Naniwadekar predict the crack based on the changes in natural frequency can handle crack in various orientations in straight pipes. Yu derived the local flexibility under different loading in homogeneous and non-homogeneous beams and used $\mathrm{p}$ version of finite element method to analyze the characteristics and dynamic response of cracked structures[1].

In this paper, local flexibility coefficients due to the crack in the vibrating screen beam is given firstly based on the previous research. Then the continuous model which is contained crack is presented for the vibration analysis and crack detection. Through numerical analysis, the location and depth of the crack can be both detected and the crack quantitative detection of vibrating screen beam can be realized. 


\section{Local flexibility of a cracked vibrating screen beam}

This paper take the circular-hollow-sectional vibrating screen beam as example to calculate the local flexibility firstly. The 3-D figure of vibrating screen beam is shown in figure 2 :

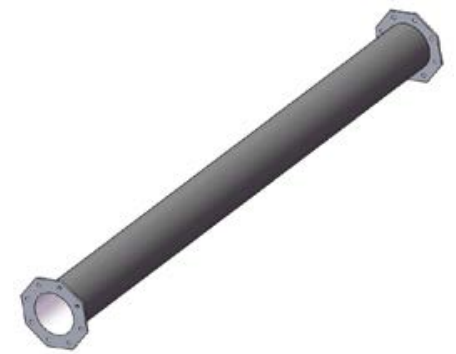

Fig. 2 Circular-hollow-sectional vibrating screen beam

D. Y. Zheng and S. C. Fan derived the local flexibility coefficients due to the existence of an open sectional crack in rectangular or circular-hollow-sectional beams[2]. This paper apply the method based on their research to present the local flexibility coefficient of the crack under the assumption that the crack is always open. The shallow open crack of vibrating screen beam is shown in figure 3 :

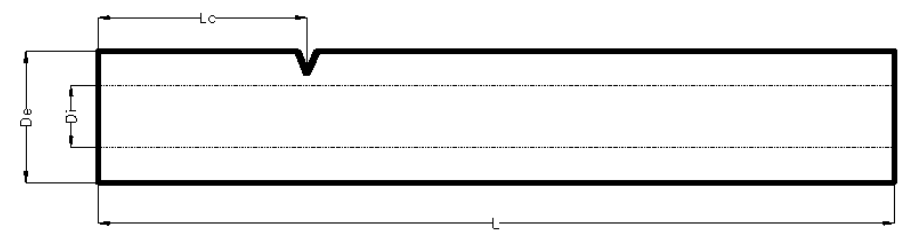

Fig. 3 Shallow crack in vibrating screen beam

$\mathrm{D}_{\mathrm{e}}$ is the external diameter of the beam and $\mathrm{D}_{\mathrm{i}}$ is the internal diameter. The cracked cross-sectional geometry is shown in figure 4 and a is the depth of a sectional crack:

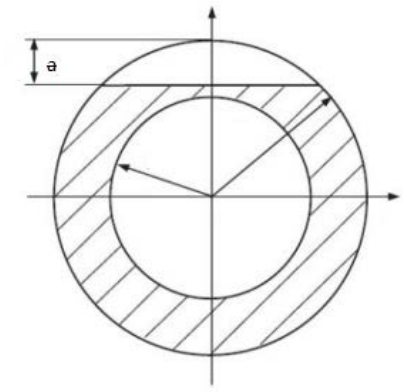

Fig. 4 Cracked cross-sectional geometry

According to the theory of linear fracture mechanics, the additional strain energy due to the existence of crack can be expressed as Eq.(1)

$$
U_{T}=\int_{A_{c}} G d A
$$

$A_{c}$ is the surface area of the crack. $G$ is a function of the energy release rate and it can be expressed as Eq.(2)

$$
G=\frac{1}{E^{\prime}}\left[\left(K_{\mathrm{I} 1}+K_{\mathrm{I} 2}+K_{\mathrm{I} 3}\right)^{2}+K_{\mathrm{II} 2}^{2}\right]
$$

$\mathrm{K}$ is the stress intensity factor. Considering the condition of the beam is bearing the bending load M, G can be expressed as Eq.(3)

$$
G=\frac{1}{E^{\prime}} K_{\mathrm{I} 1}^{2}
$$

$\mathrm{E}^{\prime}=E$ for plane-stress problem while $E^{\prime}=\mathrm{E} /\left(1-v^{2}\right)$ for plane-strain problem, in which $\mathrm{E}^{\prime}$ is Young's modulus and $v$ is the Poisson ratio.

According to the Castigliano's theorem, the displacement of the crack under the bending can be computed as Ep.(4)

$$
\mathrm{u}=\frac{d U_{T}}{d \mathrm{P}}
$$


$P$ is the load and the additional local flexibility is as Eq.(5)as follows:

$$
\mathrm{c}=\frac{d u}{d P}
$$

The derivation process will not be given in detail. We can obtain the local flexibility as Eq.(6)

$$
c=\frac{2048}{\pi E^{\prime} D_{e}^{3}\left(1-\gamma^{4}\right)^{2}} \int_{0}^{a / D_{e}} \int_{-\sqrt{x-x^{2}}}^{\sqrt{x-x^{2}}}\left(1-4 y^{2}\right)\left(x+\sqrt{\frac{1}{4}-y^{2}}-\frac{1}{2}\right) F^{2}(s) d y d x
$$

Where $\gamma=\mathrm{D}_{i} / D_{e}, \mathrm{~F}(\mathrm{~s})$ is the correction factors for stress intensity factors. The dimensionless form can be expressed as Eq.(7)

$$
c E^{\prime} D_{e}^{3}=\frac{2048}{\pi\left(1-\gamma^{4}\right)^{2}} \int_{0}^{a / D_{e}} \int_{-\sqrt{x-x^{2}}}^{\sqrt{x-x^{2}}}\left(1-4 y^{2}\right)\left(x+\sqrt{\frac{1}{4}-y^{2}}-\frac{1}{2}\right) F^{2}(s) d y d x
$$

After the calculation of this double integral we can obtain the relation between relative depth of crack and dimensionless local flexibility, which is shown in figure 5:

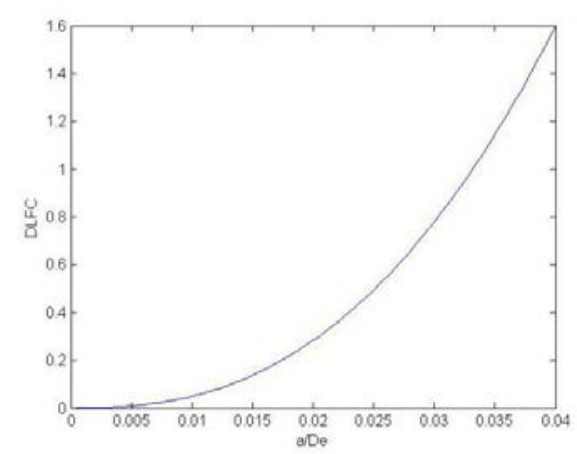

Fig. 5 Dimensionless local flexibility versus relative depth of a crack in a circular-hollow-sectional beam

\section{Vibration analysis of beam's analytical model with an open crack}

The crack of the beam can be equivalent to a spring and it's shown in Fig. 6:

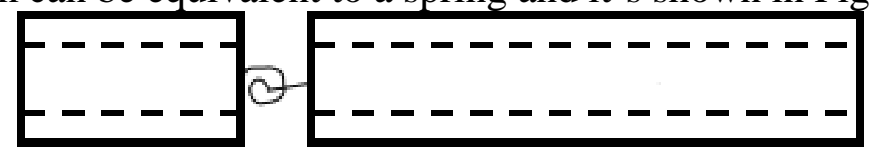

Fig. 6 Equivalent model of cracked beam

The vibration equation of beam can be expressed as follows in a well known form[3]:

$$
E I \frac{\partial^{4} y(x, t)}{\partial x^{4}}+\rho A \frac{\partial^{2} y(x, t)}{\partial t^{2}}=0
$$

where $E$ is Young's modulus. $\rho$ is material density. $I$ is the geometrical moment of inertia of the cross-section. $A$ is cross-sectional area of the beam and $x$ is the coordinate along the beam. The form of the equation's solution can be written as:

$$
y(x, t)=Y(x) \sin (\omega t)
$$

The vibration screen beam is divided into two parts due to the crack and the equation can be expressed respectively as follows:

$$
\begin{cases}\frac{d^{4} Y_{1}(x)}{d x^{4}}-\frac{\rho A \omega^{2}}{E I} Y_{1}(x)=0 & \left(0 \sim L_{c}\right) \\ \frac{d^{4} Y_{2}(x)}{d x^{4}}-\frac{\rho A \omega^{2}}{E I} Y_{2}(x)=0 & \left(L_{c} \sim L\right)\end{cases}
$$

The form of the solution can be written as: 


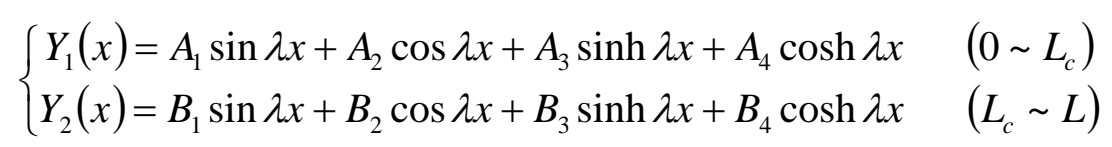

where $\lambda^{4}=\frac{\rho \mathrm{A} L^{4}}{E I} \omega^{2}, \beta=\frac{\mathrm{x}}{L}$.

Coupled with the the boundary conditions, continuity conditions, compatibility condition of the vibrating screen beam[4], we can get as Eq.(12):

\begin{tabular}{|c|c|c|c|c|c|c|c|}
\hline 0 & 1 & 0 & 1 & 0 & 0 & 0 & 0 \\
\hline 1 & 0 & 1 & 0 & 0 & 0 & 0 & 0 \\
\hline $\sin \lambda \beta$ & $\cos \lambda \beta$ & $\sinh \lambda \beta$ & $\cosh \lambda \beta$ & $-\sin \lambda \beta$ & $-\cos \lambda \beta$ & $-\sinh \lambda \beta$ & $-\cosh \lambda \beta$ \\
\hline$-\sin \lambda \beta$ & $-\cos \lambda \beta$ & $\sinh \lambda \beta$ & $\cosh \lambda \beta$ & $\sin \lambda \beta$ & $\cos \lambda \beta$ & $-\sinh \lambda \beta$ & $-\cosh \lambda \beta$ \\
\hline$-\cos \lambda \beta$ & $\sin \lambda \beta$ & $\cosh \lambda \beta$ & $\sinh \lambda \beta$ & $\cos \lambda \beta$ & $-\sin \lambda \beta$ & $-\cosh \lambda \beta$ & $-\sinh \lambda \beta$ \\
\hline$-\cos \lambda \beta$ & $\sin \lambda \beta$ & $-\sinh \lambda \beta$ & $-\cosh \lambda \beta$ & $\frac{E I}{K_{T} L} \lambda \sin \lambda \beta+\cos \lambda \beta$ & $\frac{E I}{K_{T} L} \lambda \cos \lambda \beta-\sin \lambda \beta$ & $\frac{E I}{K_{T} L} \lambda \sinh \lambda \beta+\cosh \lambda \beta$ & $-\frac{E I}{K_{T} L} \lambda \cosh \lambda \beta+\sinh \lambda \beta$ \\
\hline 0 & 0 & 0 & 0 & $\sin \lambda$ & $\cos \lambda$ & $\sinh \lambda$ & $\cosh \lambda$ \\
\hline 0 & 0 & 0 & 0 & $\cos \lambda$ & $-\sin \lambda$ & $\cosh \lambda$ & $\sinh \lambda$ \\
\hline
\end{tabular}

$\mathrm{K}_{\mathrm{T}}$ is the equivalent stiffness of the crack, and it is equal to the reciprocal of the local flexibility due to the crack. The characteristic root $\lambda$ can be get from Eq.(13), and then the natural frequency which is used for the crack detection can be calculated as follows:

$$
\omega=\left(\frac{\lambda_{\mathrm{i}}}{L}\right)^{2} \sqrt{\frac{E I}{\rho A}}
$$

Thus we can get the relationship between frequency, depth and location[5]. And the crack of vibrating screen beam can be detected quantificationally according to this relationship.

\section{Conclusions}

At present, most mining enterprises adopt the periodical maintenance or break maintenance to the key equipment. The workers check the vibration screen by eyes and this approach is not purposeful. In addition, the most important thing is some faults like cracks would be neglected sometimes due to the dusky environment. This paper introduce a continuous model of cracked vibration screen beam based on the calculation of local flexibility due to the crack. and free vibration analysis get the relation between the natural frequency and the depth, location of the crack. Then the detection of crack can be achieved by detecting the change of the natural frequency, and it make the crack quantitative detection of the vibration screen beam come true.

\section{Acknowledgments}

This work was financially supported by the National Natural Science Fund(U1361127).

\section{References}

[1] Chondros T G, Dimarogonas A D. Dynamic Sensitivity of Structures to Cracks. Journal of Vibration \& Acoustics, 111(1989)251-256.

[2] Zheng D Y, Fan S C. Vibration and stability of cracked hollow-sectional beams. Journal of Sound \& Vibration, 267(2003)933-954.

[3] G.M.Dong, J.Chen, J.Zou. Parameter identification of a rotor with an open crack. European Journal of Mechanics - A/Solids, 23(2004)325-333.

[4] Zheng D Y, Kessissoglou N J. Free vibration analysis of a cracked beam by finite element method. Journal of Sound \& Vibration, 273(2004)457-475.

[5] Papadopoulos C A. The strain energy release approach for modeling cracks in rotors: A state of the art review. Mechanical Systems \& Signal Processing, 22(2008)763-789. 